\title{
L-asparaginase encapsulation into asymmetric permeable polymersomes
}

\author{
Cecilia Z. Bueno, ${ }^{\dagger, \ddagger}$ Alexsandra C. Apolinário ${ }^{\dagger, \|, \ddagger}$, Aroa Duro-Castano ${ }^{\ddagger}$, Alessandro \\ Poma ${ }^{\ddagger},+$, Adalberto Pessoa-Jr, ${ }^{\dagger}$ Carlota O. Rangel-Yagui, ${ }^{*}{ }^{\dagger}$ Giuseppe Battaglia ${ }^{*}$,,$\S$, \\ $\perp$ \\ † Department of Biochemical and Pharmaceutical Technology, University of São Paulo, 05508-000, \\ São Paulo, Brazil \\ I| Department of Pharmacology, University of São Paulo, 05508-000, São Paulo, Brazil \\ ‡ Department of Chemistry, University College London, WC1H 0AJ, London, United Kingdom \\ $\dashv$ Eastman Dental Institute, University College London, WC1X 8LD, London, United Kingdom \\ $\S$ Institute for Bioengineering of Catalonia (IBEC), 08028, Barcelona Institute for Science and \\ Technology (BIST), 08036, Barcelona, Spain \\ $\perp$ Catalan Institution for Research and Advanced Studies, (ICREA), 08010, Barcelona, Spain
}

\section{S1: Materials and Methods}

\section{Materials}

L-asparaginase was purchased from ProSpec-Tany TechnoGene Ltd. (Ness Ziona, Israel). PEO $_{16^{-}}$ $\mathrm{PBO}_{22}(\mathrm{MW}=1910 \mathrm{Da})$ was purchased from Advanced Polymer Materials Inc.. L-asparagine, phosphotungstic acid, sepharose 4B (45-165 $\mu \mathrm{m}$ bead diameter), Trizma $\mathrm{HCl}$, Trizma base, ammonium sulphate, trichloroacetic acid and Nessler reagent were purchased from Sigma-Aldrich. Phosphate buffered saline (PBS) tablets, trifluoroacetic acid, uranyl acetate, chloroform and methanol were from Fisher Scientific. Methanol for HPLC was from VWR. Micro-BCA kit was purchased from Thermo Scientific and Aspartate assay kit was from Abcam.

\section{Copolymer synthesis}

$\mathrm{PMPC}_{25}-\mathrm{PDPA}_{70}$ diblock copolymer (MW=21600 Da) is synthesized using atom transfer radical polymerization (ATRP) in the presence or in the absence of Cy5 dye (Cyanine5) as described elsewhere $1-3$.

\section{${ }^{1}$ H-Nuclear Magnetic Resonance (NMR)}

NMR spectrum of PMPC-PDPA polymer is recorded at $27^{\circ} \mathrm{C}(300 \mathrm{~K})$ on a Bruker Avance III 600 (USA) spectrometer. Data is processed with the software Mestrenova 9.0. Samples were prepared at 10 $\mathrm{mg} / \mathrm{mL}$ concentration in a mixture $3 / 1(\mathrm{v} / \mathrm{v})$ of deuterated chloroform and deuterated methanol. The copolymer NMR analysis is shown in Supporting Information S2. 


\section{Polymersome preparation}

Three different polymersome formulations are prepared: 100\% PMPC-PDPA (100:0), 100\% PEOPBO (0:100) and 90\% PMPC-PDPA/10\% PEO-PBO (90:10). In the formulations containing PMPC-PDPA, the copolymer is mixed with $20 \%(\mathrm{~mol} / \mathrm{mol})$ of PMPC-PDPA fluorescently labelled with Cy5 dye (Cyanine5) to facilitate the visualization of the systems during the purification processes. All polymersome formulations are prepared by the film hydration method ${ }^{1}$. The initial polymer solutions are prepared in chloroform/methanol 2:1 (v/v) at a total polymer concentration of $10 \mathrm{mg} / \mathrm{mL}$. The solutions are filtered with $0.2 \mu \mathrm{m}$ pore membranes into glass vials and dried inside a vacuum oven for $24 \mathrm{~h}$ at $30^{\circ} \mathrm{C}$. Afterwards, PBS at pH 7.4 previously filtered with $0.2 \mu \mathrm{m}$ pore membranes is added to the flasks, resulting in a final polymer concentration of $5 \mathrm{mg} / \mathrm{mL}$. Polymersome systems constituted by PMPC-PDPA copolymer alone or in combination with PEO-PBO are magnetically stirred for 4 weeks at 2000 RPM and room temperature. After this period, when the suspensions become turbid, the liquid is collected into microcentrifuge tubes and centrifuged at $1000 \mathrm{RCF}$ for $5 \mathrm{~min}$ to remove non-assembled polymer. Polymersome systems constituted only by PEO-PBO copolymer are stirred for 12 hours at 2000 RPM and room temperature. Then, the systems are manually extruded 15 times with a $0.4 \mu \mathrm{m}$ extrusion membrane followed by 15 times with a $0.2 \mu \mathrm{m}$ extrusion membrane (Avanti mini extruder - Avanti Polar Lipids ${ }^{\circledR}$ ).

\section{L-asparaginase encapsulation}

ASNase is encapsulated through electroporation (Electroporator 2510, Eppendorf ${ }^{\circledR}$ ). First, $400 \mu \mathrm{L}$ of ASNase solution ( $5 \mathrm{mg} / \mathrm{mL}$ ) is mixed with $400 \mu \mathrm{L}$ of polymersomes suspension in an $800 \mu \mathrm{L}(4 \mathrm{~mm})$ electroporation cuvette. Electroporation is carried out at an initial voltage of $2500 \mathrm{~V}$ and resulting voltage of around $700 \mathrm{~V}$. The number of pulses employed is 10 or 20 with an interval of $60 \mathrm{~s}$ between each pulse.

\section{Polymersomes purification}

The systems are purified by size-exclusion chromatography (SEC) ${ }^{4}$ to remove non-encapsulated enzyme. Polymersome suspensions $(700 \mu \mathrm{L})$ are eluted through a Sepharose 4B column $(1 \mathrm{~cm}$ in internal diameter and $20 \mathrm{~cm}$ in height), using PBS filtered with $0.2 \mu \mathrm{m}$ pore size membranes as the mobile phase. The eluted fractions $(200 \mu \mathrm{L})$ are collected in a transparent 96 well plate and DLS measurements confirmed the fractions corresponding to pure polymersomes. Micro-BCA assay is used to confirm the separation between vesicles and free enzyme.

\section{Polymersomes characterization}

\section{Dynamic light scattering (DLS)}

Dynamic light scattering (DLS) is performed in a DLS Nanosizer DTS0012 (Malvern ${ }^{\circledR}$ ) operating with a He-Ne laser $(633 \mathrm{~nm})$ and a detector set at $173^{\circ}$. The equipment is set with the following parameters: equilibration time of $60 \mathrm{~s}, 3$ runs with automatic number of repetitions and temperature of 
$20^{\circ} \mathrm{C}$. Before DLS analysis, samples are diluted with filtered PBS at 1:20 and transferred to polystyrene cuvettes.

\section{Transmission electron microscopy (TEM)}

Before TEM analysis, polymersome samples are mounted on copper grids covered with carbon film (C400Cu100, EM Resolutions) previously glow-discharged at $30 \mathrm{~mA}$ for $45 \mathrm{~s}$. A small volume (5 $\mu \mathrm{L})$ of sample diluted in Milli-Q water (approximately $0.1-0.2 \mathrm{mg} / \mathrm{mL}$ ) is placed over the grid for 1 minute. The grid is then blotted with filter paper. Samples containing PMPC-PDPA were placed over a $20 \mu \mathrm{L}$ drop of phosphotungstic acid (PTA) at $7.5 \mathrm{mg} / \mathrm{mL}$ and pH 7.4 for 3 sec. Samples consisting of only PEO-PBO are stained with $5 \mu \mathrm{L}$ of uranyl acetate solution $(2 \% \mathrm{w} / \mathrm{w})$ for 1 minute. The grids are then blotted with filter paper and carefully dried using a vacuum pump. TEM analysis is carried on an electron microscope JEOL (JEM-2100) or JEOL-1400 Plus.

\section{High-performance liquid chromatography}

Reversed-phase high-performance liquid chromatography (RP-HPLC) is employed to measure the amount of encapsulated enzyme after breaking the polymersomes containing PMPC-PDPA under acidic pH. This analysis is not employed for 100\% PEO-PBO samples. Firstly, PBS at pH 2.5 is added dropwise to the polymersomes suspension until reaching $\mathrm{pH}$ 6.0, causing the protonation of the tertiary amino groups of the PDPA chains (pKa=6.4) and the consequent polymersome disassembly ${ }^{5}$. Afterwards, $100 \mu \mathrm{L}$ of the disrupted polymersomes samples are injected in the equipment (Dionex Ultimate 3000 HPLC) and eluted through a C18 column (Phenomenex Jupiter $5 \mu \mathrm{m}, 300 \AA$, $4.60 \mathrm{~mm} \times 250 \mathrm{~mm}$ column) at $1 \mathrm{~mL} / \mathrm{min}$ constant flow. A gradient of an aqueous solution of trifluoroacetic acid 0.05\% v/v (TFA) (eluent A) and methanolic solution of TFA $0.05 \% \mathrm{v} / \mathrm{v}$ (eluent B) is used to run the samples through the column. The gradient consisted of $5 \%$ eluent B from 0 to 20min, 100\% eluent B from 20 to $25 \mathrm{~min}$ and $5 \%$ eluent B from 25 to $31 \mathrm{~min}^{1}$.

PMPC-PDPA is detected by UV-VIS absorbance at $220 \mathrm{~nm}$ and ASNase is detected by fluorescence (excitation wavelength: $280 \mathrm{~nm}$; emission wavelength: $320 \mathrm{~nm}$ ). The polymer and the enzyme are quantified according to the peak area using previously measured standard curves. The resulting encapsulation data are calculated as demonstrated by Wang et al. (2012) ${ }^{6}$.

\section{Bicinchoninic acid assay (Micro-BCA)}

Micro-BCA assay ${ }^{7}$ is used to measure the amount of total protein in samples containing ASNase according to BSA (bovine serum albumin) standard curves. The assay is prepared by mixing $150 \mu \mathrm{L}$ of working reagent and $150 \mu \mathrm{L}$ of sample in a transparent 96 well plate. Samples are incubated for 2 hours at $37^{\circ} \mathrm{C}$, cooled down to room temperature and absorbance is measured at $562 \mathrm{~nm}$ in a plate reader. Experiments are performed in triplicate.

\section{Nessler assay}

ASNase activity is quantified according to the Nessler assay ${ }^{8}$, which consists of a colorimetric determination of the ammonia released in the cleavage of Asn by ASNase. For low enzyme concentrations, 
the conventional Nessler protocol is modified to increase the incubation time and the ratio between the volume of the sample and the volume of the reagents, therefore improving the sensitivity of the method.

Firstly, $50 \mu \mathrm{L}$ of $50 \mathrm{mM}$ Tris Buffer (pH 8.6 at $\left.37^{\circ} \mathrm{C}\right), 200 \mu \mathrm{L}$ of water and $50 \mu \mathrm{L}$ of Asn solution (25 $\mathrm{mg} / \mathrm{mL}$ in water) are mixed and incubated at $37^{\circ} \mathrm{C}$ for $5 \mathrm{~min}$. Then $50 \mu \mathrm{L}$ of polymersome sample is added and the tubes are incubated at $37^{\circ} \mathrm{C}$ for 2 hours. The reaction is then interrupted by the addition of $50 \mu \mathrm{L}$ of $1.5 \mathrm{M}$ trichloroacetic acid (TCA). Afterwards, $100 \mu \mathrm{L}$ of each tube is mixed with $500 \mu \mathrm{L}$ of ultrapure water, followed by the addition of $250 \mu \mathrm{L}$ of Nessler's reagent. After 1 min of reaction, $200 \mu \mathrm{L}$ of each sample is transferred to a 96 well plate and the absorbance at $436 \mathrm{~nm}$ is measured in a plate reader. Polymersome systems without encapsulated ASNase are used as blanks. A standard curve is plotted using $\left(\mathrm{NH}_{4}\right)_{2} \mathrm{SO}_{4}$ instead of ASNase.

The number of enzyme units (U) is calculated based on the information that one unit of ASNase is the amount of enzyme which catalyses the formation of $1 \mu \mathrm{mol}$ ammonia per minute at $37^{\circ} \mathrm{C}$. All measurements are performed in triplicate.

\section{Aspartate Assay}

The Aspartate assay kit (Abcam) is used to determine aspartate concentration in polymersomes systems, after incubation with Asn. In this assay, aspartate is converted to pyruvate, which is oxidized with the conversion of a probe into a fluorescent species proportional to the amount of aspartate in the samples.

Firstly, $200 \mu \mathrm{L}$ of Asn solution (25 mg/mL) and $100 \mu \mathrm{L}$ of polymersome samples with or without ASNase are mixed and incubated at $37^{\circ} \mathrm{C}$ for 30 minutes. Then, the mixture is diluted 500 times with PBS and $50 \mu \mathrm{L}$ is added to $50 \mu \mathrm{L}$ of the assay's reaction mix in 96 well plates. A standard curve is prepared with an aspartate standard. The plate is incubated for $30 \mathrm{~min}$ at room temperature and fluorescence at Ex/Em $535 \mathrm{~nm} / 587 \mathrm{~nm}$ is measured in a plate reader. The experiments are performed in triplicate.

\section{Adequacy of the Aspartate assay based on the obtained results}

To verify the adequacy of the assay to measure aspartate release by ASNAse-encapsulating polymersomes, we consider the possibility of aspartic acid formation inside the vesicle and consequent disruption due to $\mathrm{pH}$ decrease. Thus, to prove that aspartic acid is not causing the dissociation of the vesicles, we calculate the maximum amount of aspartate that would form if all the asparagine is hydrolysed in the presence of the encapsulated enzyme during the aspartate assay.

We first calculate the amount of asparagine added to $100 \mu \mathrm{L}$ of polymersomes, which is equal to $3.8 \times 10^{-5} \mathrm{~mol}$, or $380 \mathrm{mM}$. Since $1 \mathrm{~mol}$ of asparagine forms $1 \mathrm{~mol}$ of aspartate, one should obtain $380 \mathrm{mM}$ of aspartate for $100 \%$ reaction. As can be observed in Figure 4B, only a maximum of around $8 \mathrm{mM}$ is detected at the end of the assay.

We perform a similar calculation for the Nessler assay. In this case, if reaction is complete, one should obtain $190 \mathrm{mM}$ of $\mathrm{NH}_{3}$. As can be observed in Figure 4A, the maximum amount of ammonia detected is of around $17 \mathrm{mM}$.

These results provide some evidence that the amount of acid inside the polymersomes is not sufficient to disrupt the vesicles. 


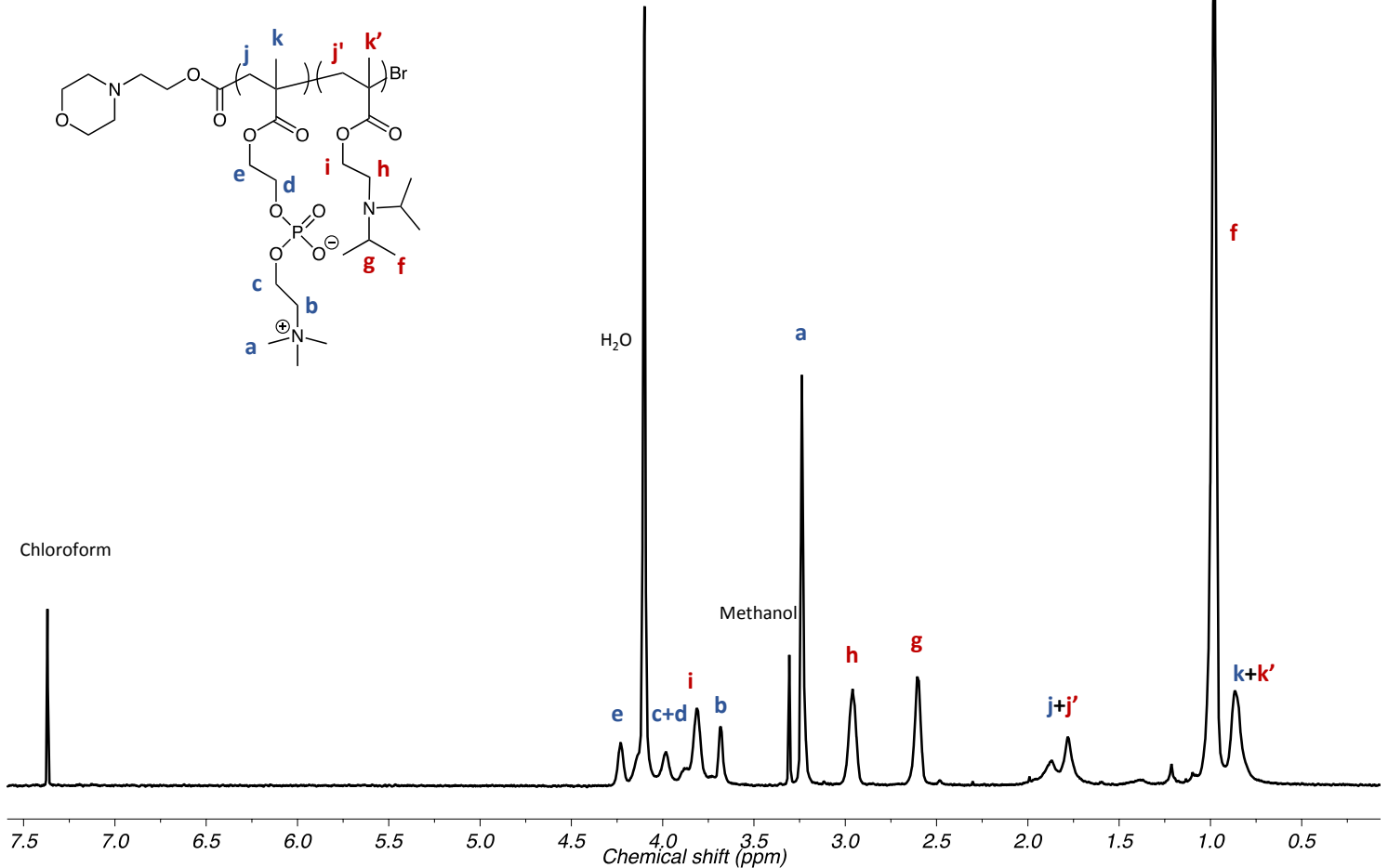

Figure S1: ${ }^{1} \mathrm{H}$ NMR Spectrum of $\mathrm{PMPC}_{25}-\mathrm{PDPA}_{70}$. 


\section{S3: Polymersomes stability after 2 months (DLS analysis)}

The results shown here were used only for the stability studies and were not further characterized. The results shown in the main text are referent to other systems.
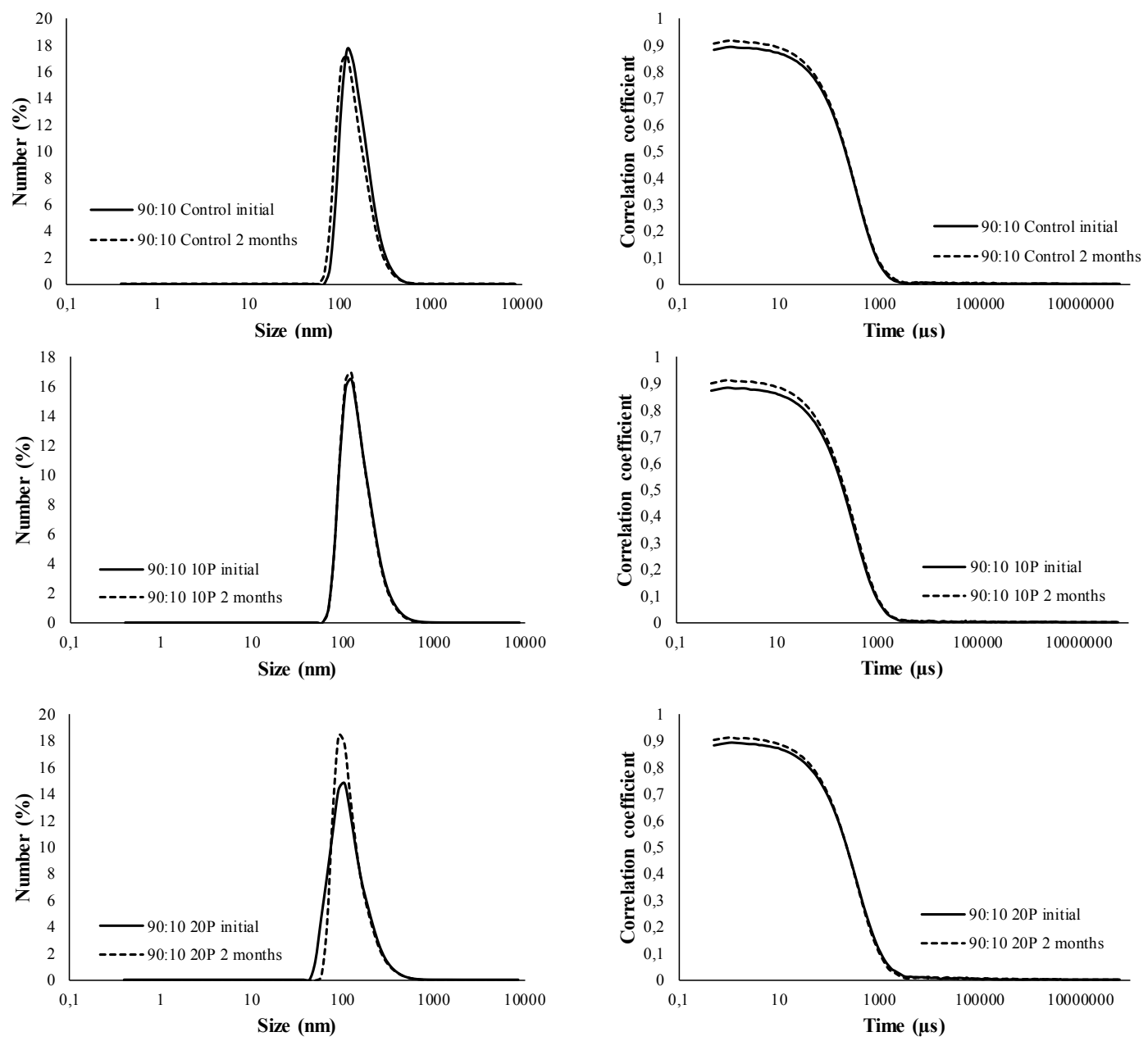

Figure S2: Dynamic light scattering measurements of asymmetric polymersomes systems (90:10) right after preparation and purification by size exclusion chromatography and after 2 months storage. 

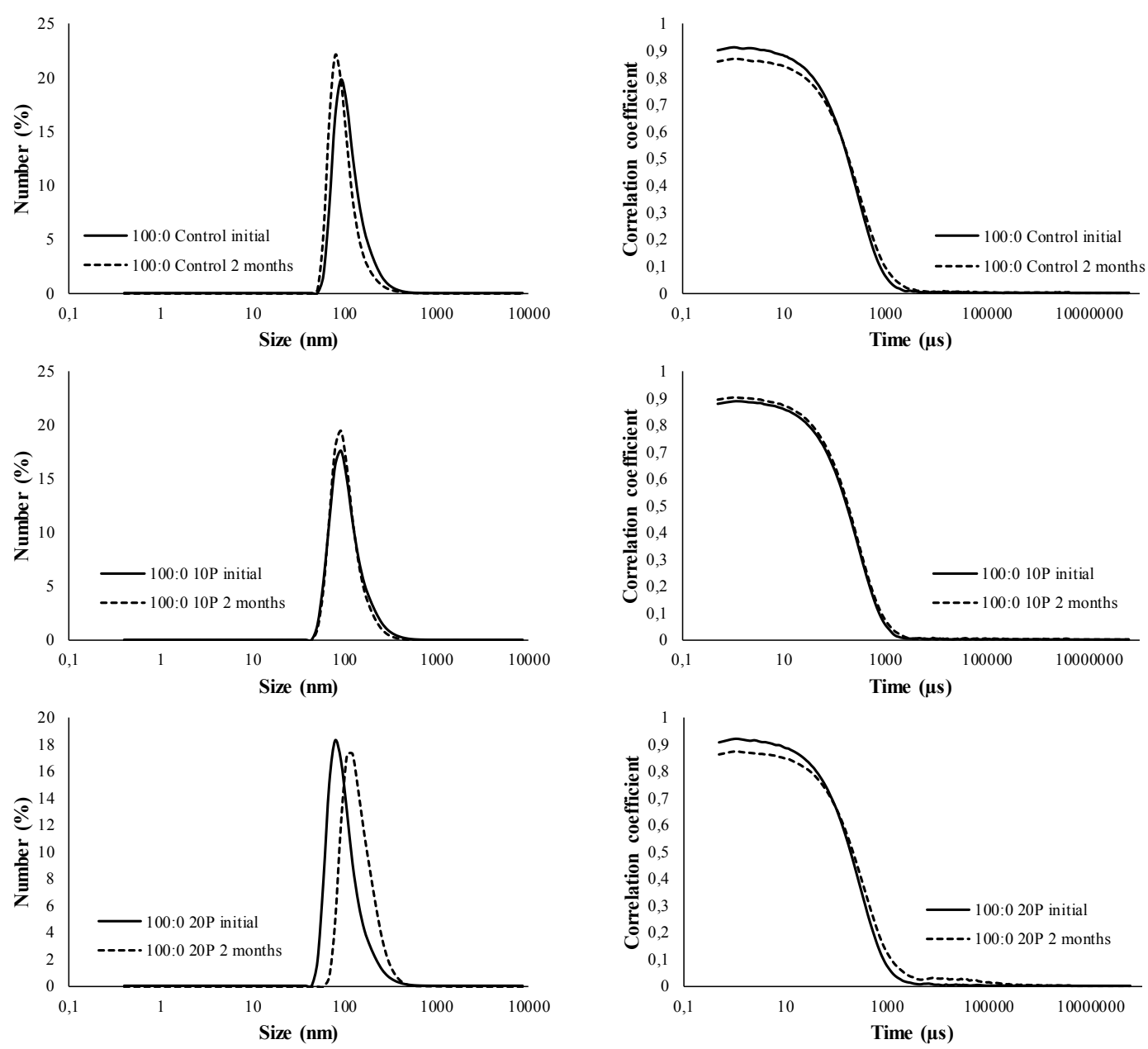

Figure S3: Dynamic light scattering measurements of symmetric polymersomes systems (100:0) right after preparation and purification by size exclusion chromatography and after 2 months storage. 


\section{S4: PEO-PBO polymersomes characterization}
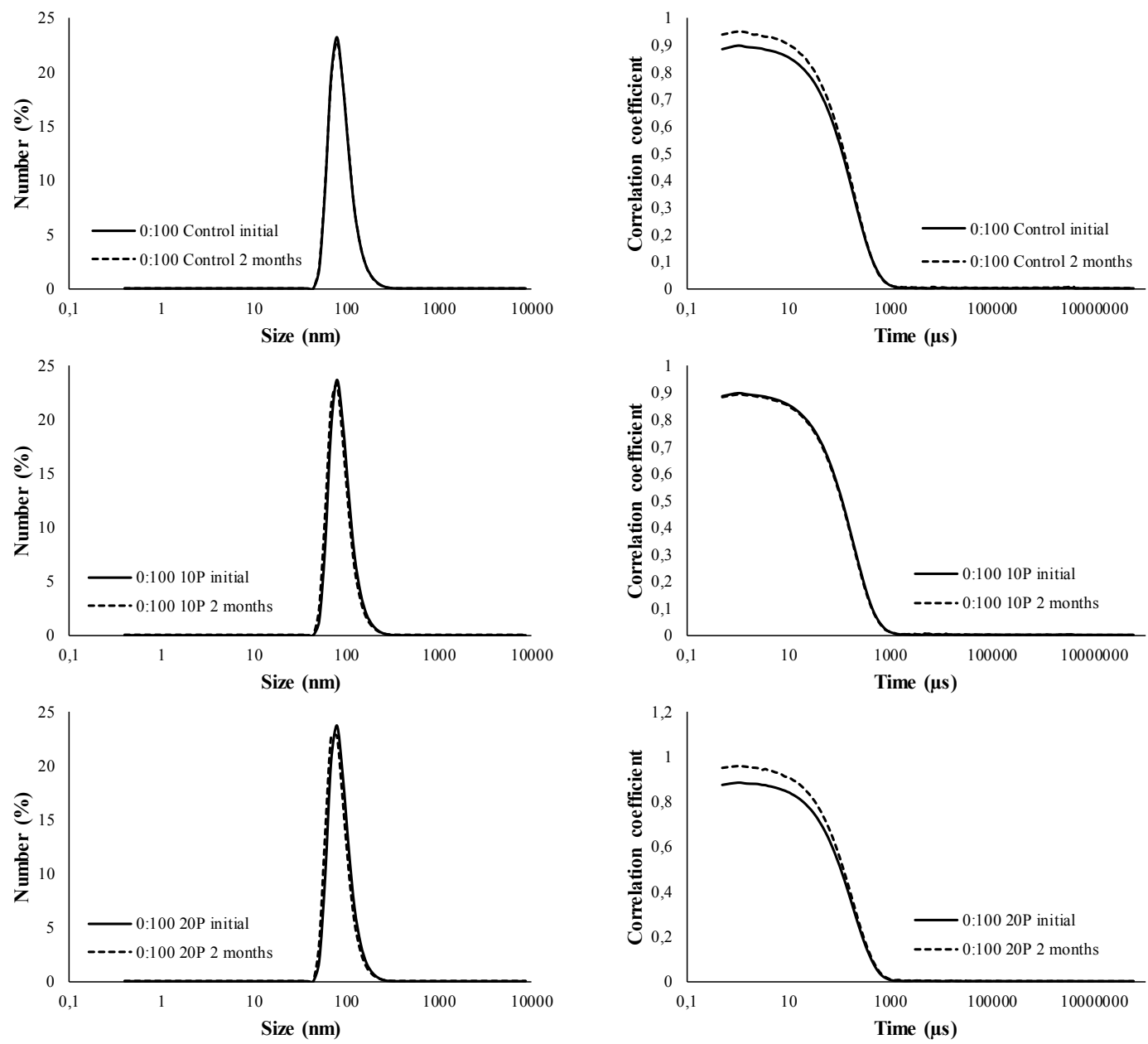

Figure S4: Dynamic light scattering measurements of pure PEO-PBO polymersomes (0:100) right after purification by size exclusion chromatography and after 2 months storage.
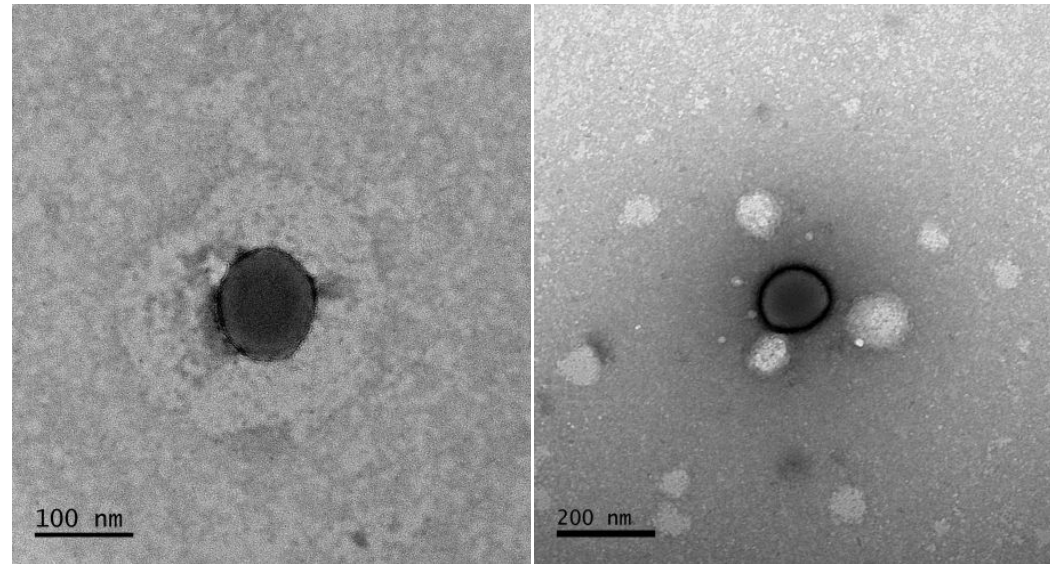

Figure S5: TEM micrographs of symmetric PEO-PBO (0:100) polymersomes. 


\section{S5: L-asparaginase purification by SEC (Micro BCA analysis)}

Data shown in Table S1 were obtained after electroporating and purifying the enzyme by SEC, substituting the polymersomes system by PBS (pH 7.4). Purified fractions were collected in a 96-well plate from 5 to $20 \mathrm{~min}$ and analyzed by Micro-BCA assay.

Table S1: ASNase concentrations in each fraction eluted from SEC purification for solutions electroporated with 10 and 20 pulses. Highlighted lines indicate the average time in which polymersomes leave the column.

\begin{tabular}{|c|c|c|}
\hline \multirow{2}{*}{ Time (min) } & \multicolumn{2}{|c|}{ [ASNase] $(\boldsymbol{\mu g} / \mathbf{m L})$} \\
\cline { 2 - 3 } & $\mathbf{1 0}$ pulses & $\mathbf{2 0 ~ p u l s e s}$ \\
\hline $5-6.7$ & $0.0 \pm 0.0$ & $0.1 \pm 0.1$ \\
\hline $6.7-8.4$ & $0.2 \pm 0.2$ & $0.0 \pm 0.0$ \\
\hline $\mathbf{8 . 4}-\mathbf{1 0 . 1}$ & $\mathbf{0 . 1} \pm \mathbf{0 . 1}$ & $\mathbf{0 . 0} \pm \mathbf{0 . 0}$ \\
\hline $\mathbf{1 0 . 1}-\mathbf{1 1 . 8}$ & $\mathbf{0 . 1} \pm \mathbf{0 . 1}$ & $\mathbf{0 . 0} \pm \mathbf{0 . 0}$ \\
\hline $\mathbf{1 1 . 8}-\mathbf{1 3 . 5}$ & $\mathbf{0 . 0} \pm \mathbf{0 . 0}$ & $\mathbf{0 . 3} \pm \mathbf{0 . 0}$ \\
\hline $13.5-15.2$ & $0.1 \pm 0.0$ & $1.2 \pm 0.0$ \\
\hline $15.2-16.9$ & $1.1 \pm 0.0$ & $2.3 \pm 0.0$ \\
\hline $16.9-18.6$ & $3.7 \pm 0.0$ & $3.4 \pm 0.3$ \\
\hline $18.6-20.3$ & $8.4 \pm 0.1$ & $4.8 \pm 0.1$ \\
\hline
\end{tabular}

As can be seen, most part of the enzyme leave at the final fractions, proving the effectiveness of SEC purification to remove non-encapsulated enzyme. However, a small amount of ASNase leaves the column at the same time as the polymersomes, probably due to larger protein aggregates. 


\section{S6: Encapsulation results calculations}

Polymersomes loading capacity was expressed as the average number of ASNase molecules in each polymersome $\left(L_{N}\right)$ and calculated from DLS and HPLC data as described by Wang et al. (2012) and Joseph et al. (2017).

$$
L_{N}=\frac{N_{b}}{N_{p}}
$$

where $N_{b}$ is the number of ASNase molecules and $N_{p}$ is the total number of polymersomes in the purified system. $N_{b}$ was calculated according to the equation:

$$
N_{b}=\frac{M_{b}}{M_{w b}} N_{A}=\frac{C_{b} V_{s}}{M_{w b}} N_{A}
$$

where $M_{b}$ is the mass of ASNase, $M_{w b}$ is the ASNase molecular weight, $C_{b}$ is the ASNase concentration, $V_{s}$ is the volume of the collected solution after purification and $N_{A}$ is the Avogadro's number.

The total number of polymersomes in the purified system was calculated as:

$$
N_{P}=\sum_{1}^{n} N_{p}^{i}
$$

where $N_{p}^{i}$ corresponds to the number of polymersomes for each population of size, which was calculated as the following:

$$
N_{p}^{i}=\frac{M_{c}}{M_{w c} \times N_{a}^{i}} \times N_{A} \times R^{i}=\frac{C_{c} \times V_{s}}{M_{w c} \times N_{a}^{i}} \times N_{A} \times R^{i}
$$

where:

$M_{c}$ is the mass of polymersomes after purification by SEC;

$M_{w c}$ is the polymer's molecular weight;

$N_{A}$ is the Avogadro's Number;

$C_{c}$ is the copolymer concentration after purification;

$V_{s}$ is the volume of solution after purification;

$R^{i}$ is the number of polymersomes for each population of size obtained by DLS.

$N_{a}^{i}$ is the aggregation number of the copolymers and is related to the polymersomes core volume:

$$
N_{a}^{i}=\frac{\rho_{P D P A} \cdot V_{P D P A}^{i}}{M_{W P D P A}} \times N_{A}
$$

where:

$\rho_{P D P A}$ is the PDPA block density;

$M_{W P D P A}$ is the PDPA block molecular weight;

$V_{P D P A}^{i}$ is the PDPA block volume in a single polymersome and is expressed by: 


$$
V_{P D P A}^{i}=\frac{4}{3} \pi\left[(r-d)^{3}-(r-d-l)^{3}\right]
$$

(Equation S6)

where:

$r$ is the polymersome radius

$d$ is the hydrophilic brush length

$l$ is the hydrophobic thickness 


\section{S7: Verification of ASNase encapsulation (HPLC analysis)}

This experiment was performed with pure PMPC-PDPA polymersomes (100:0) as described in the main text. The protein content based on polymersomes disruption is shown in Table S2.

Table S2: ASNase encapsulation parameters detected for the electroporated enzyme mixed with nonelectroporated polymersomes.

\begin{tabular}{|c|c|c|c|c|}
\hline Sample & $\begin{array}{c}\text { Actual } \\
\text { loading/theoretical } \\
\text { loading (mg/mg) }\end{array}$ & $\begin{array}{c}\text { Polymersome } \\
\text { production } \\
\text { efficiency (\%) }\end{array}$ & $\begin{array}{c}\text { Encapsulation } \\
\text { efficiency (\%) }\end{array}$ & $\begin{array}{c}\text { Loading } \\
\text { capacity } \\
\text { (n/Psome) }\end{array}$ \\
\hline 10 pulses & 6.7 & 44.8 & 1.0 & 54.4 \\
\hline 20 pulses & 4.9 & 43.9 & 0.7 & 36.8 \\
\hline
\end{tabular}

Therefore, non-electroporated polymersomes purified together with electroporated enzyme had smaller loading efficiencies when compared to the electroporated polymersomes. Still, there is some detectable enzyme that must be accounted for as an experimental error. 


\section{Additional References}

1. Joseph A, Contini C, Cecchin D, et al. Chemotactic synthetic vesicles: Design and applications in blood-brain barrier crossing. Sci Adv 2017; 3: e1700362.

2. Murdoch C, Reeves KJ, Hearnden V, et al. Internalization and biodistribution of polymersomes into oral squamous cell carcinoma cells in vitro and in vivo. Nanomedicine 2010; 5: 1025-1036.

3. Massignani M, LoPresti C, Blanazs A, et al. Controlling Cellular Uptake by Surface Chemistry, Size, and Surface Topology at the Nanoscale. Small 2009; 5: 2424-2432.

4. Robertson JD, Rizzello L, Avila-Olias M, et al. Purification of Nanoparticles by Size and Shape. Sci Rep 2016; 6: 27494

5. Lomas H, Du J, Canton I, et al. Efficient Encapsulation of Plasmid DNA in pH-Sensitive PMPCPDPA Polymersomes: Study of the Effect of PDPA Block Length on Copolymer-DNA Binding Affinity. Macromol Biosci 2010; 10: 513-530.

6. Wang L, Chierico L, Little D, et al. Encapsulation of Biomacromolecules within Polymersomes by Electroporation. Angew Chemie Int Ed 2012; 51: 11122-11125.

7. Smith PK, Krohn RI, Hermanson GT, et al. Measurement of protein using bicinchoninic acid. Anal Biochem 1985; 150: 76-85.

8. IMADA A, IGARASI S, NAKAHAMA K, et al. Asparaginase and Glutaminase Activities of Microorganisms. J Gen Microbiol 1973; 76: 85-99. 